This item was submitted to Loughborough's Research Repository by the author.

Items in Figshare are protected by copyright, with all rights reserved, unless otherwise indicated.

\title{
A tunable nanopore sensor for the detection of metal ions using translocation velocity and biphasic pulses
}

PLEASE CITE THE PUBLISHED VERSION

http://dx.doi.org/10.1039/C6NR07224K

PUBLISHER

Royal Society of Chemistry

VERSION

AM (Accepted Manuscript)

\section{PUBLISHER STATEMENT}

This work is made available according to the conditions of the Creative Commons Attribution-NonCommercialNoDerivatives 4.0 International (CC BY-NC-ND 4.0) licence. Full details of this licence are available at: https://creativecommons.org/licenses/by-nc-nd/4.0/

\section{LICENCE}

CC BY-NC-ND 4.0

\section{REPOSITORY RECORD}

Mayne, Laura, Steven Christie, and Mark Platt. 2016. "A Tunable Nanopore Sensor for the Detection of Metal Ions Using Translocation Velocity and Biphasic Pulses”. Loughborough University. https://hdl.handle.net/2134/23099. 
A tunable nanopore sensor for the detection of metal ions using translocation velocity and biphasic pulses.

L. J. Mayne, ${ }^{a}$ S. D. R. Christie ${ }^{\mathrm{a}, \mathrm{b}^{*}}$ and M. Platt ${ }^{\mathrm{a}^{*}}$

a. Department of Chemistry, Loughborough University, Loughborough, LE11 3TU, United Kingdom.

Email: m.platt@lboro.ac.uk; Tel: +441509 222573

b. Email: S.D.Christie@lboro.ac.uk 


\begin{abstract}
A Tunable Resistive Pulse Sensor utilising a Polyurethane nanopore, has been used to characterise nanoparticles as they traverse the pore opening. Here we demonstrate that the translocation speed, conductive and resistive pulse magnitude, can be used to infer the surface charge of a nanoparticle, and act as a specific transduction signal for the binding of metal ions to ligands on the particles surface. Surfaces of silica nanoparticles were modified with a ligand to demonstrate the concept, and used to extract copper (II) ions $\left(\mathrm{Cu}^{2+}\right)$ from solution. By tuning the $\mathrm{pH}$ and ionic strength of the solution, a biphasic pulse, a conductive followed by a resistive pulse is recorded. Biphasic pulses are becoming a powerful way to characterise materials, and provide an insight into the translocation mechanism, and here we present their first use to detect the presence of metal ions in solution. We demonstrate how combinations of translocation speed and/ or biphasic pulse behaviour are used to detect $\mathrm{Cu}^{2+}$ with quantitative responses across a range of $\mathrm{pH}$ and ionic strengths. Using a generic ligand this assay allows a clear signal for $\mathrm{Cu}^{2+}$ as low as $1 \mathrm{ppm}$ with short 5 minute incubation time, and capable of measuring 10 ppm $\mathrm{Cu}^{2+}$ in the presence of 5 other ions. The method has potential for monitoring heavy metals in biological and environmental samples.
\end{abstract}

Key Words: Nanopore, Tunable Resistive Pulse Sensing, Sensor, biphasic pulses. 


\section{Introduction}

Nanomaterials designed for the extraction and/or detection of heavy metal ions in solution have gained considerable interest in recent years. Such materials can include carbon nanotubes, ${ }^{1,2}$ nanorods $^{3}$ and nanoparticles of copper $^{4}$, gold, ${ }^{5}$ magnetic, ${ }^{6}$ and silica. ${ }^{7}$ These systems can be designed to produce a signal upon binding to the metal ion or integrated into detection platforms such as atomic adsorption, ${ }^{8-11}$ electrochemical assays, ${ }^{1,12,13}$ fluorescence $^{4,14}$ and colorimetric sensors $^{15,16}$. Whilst each technology platform has its merits, not all are portable, simple to use/interpret and can require expensive equipment and/or additional gas/carrier liquids.

Nanopores are an emerging sensor technology that have been used for single molecule analysis. ${ }^{17,18}$ The study of analyte translocations through a pore is known collectively as resistive pulse sensing (RPS), and the technique has been used to characterise proteins, inorganic ions, colloids and nanoparticles. ${ }^{19-21}$ RPS has been used to study the translocations of nanoparticles as a way to determine surface charge ${ }^{22-28}$, and biological nanopores have even been modified to detect $\mathrm{Cu}^{2+}, \mathrm{Hg}^{2+}$ ions with impressive detection limits, but due to their biological components have a limited $\mathrm{pH}$ working range. ${ }^{29,30}$ A recent adaptation to RPS uses a tunable elastomeric pore termed tunable resistive pulse sensing (TRPS) allowing further versatility as the pore can be stretched in real time to suit the sample. ${ }^{31}$ As with all RPS sensors the only consumable is the tunable pore, eliminating the need for a carrier gas, fluidics, or optics. TRPS has been validated to accurately determine the concentration, size and surface charge of dispersed inorganic particles ${ }^{24,32-37}$ as well as the concentration of a range of biological analytes, which have been reviewed previously. $^{38,39}$

Conical nanopores, as used within TRPS, exhibit ionic rectification properties. ${ }^{40-43}$ The charge on the pore wall creates areas of ion accumulation and depletion within the pore depending on the applied polarity. ${ }^{42,44-46}$ This leads to the current being higher at one voltage compared to the voltage of opposite polarity, often expressed as the rectification ratio. In the simplest setup i.e. the absence of any nanoparticles, the rectification ratio has been used to measure the concentration of metal ions as they interact with the pore wall itself. ${ }^{47}$ However these assays are limited to one analyte and longer assay times. The use of nanoparticle based systems may allow for multiplexed assays and faster reaction times. ${ }^{32,33}$ The addition of charged particles into a pore exhibiting rectification behaviour is however more complex. White and co-workers demonstrated that a negatively charged particle passing through a pore with a negative surface charge can create a conductive pulse prior to the resistive pulse at a negative polarity. ${ }^{48}$ Conversely a positive particle under the same conditions will only generate a resistive pulse, due to an increased ion depletion effect. Other groups study this by demonstrating how the resistive pulse magnitude is also dependent on particle surface charge. ${ }^{28,49}$

The nature of the rectification ratio and pulse magnitudes has only recently been studied in simple electrolyte solutions, and here we exploit these properties by designing the surface chemistry of a nanoparticle to preferentially bind to a metal ion 
in solution. The selective nature of the ligand then induces a change in the double layer structure in the presence of certain metal ions. We demonstrate that the waveshape and the velocity of the particle can be related to the particles surface charge and we use this to detect the metal ion on the surface of the nanoparticle. For this work, we focus on copper which is a heavy metal monitored in drinking water, and set at a $2 \mathrm{ppm}$ limit by the World Health Organisation (WHO). ${ }^{50}$ Copper is an essential transition metal in biology, from cofactors in metabolic reactions, ${ }^{51}$ free radical scavengers, to cell receptors and reporters. Its poor regulation can effect liver damage and be a biomarker for dementia. ${ }^{52}$

This assay uses Si nanoparticles, Figure 1a, that are modified with (3Aminopropyl)triethoxysilane, APTES, Figure 1b, a ligand known to have a preferential binding to $\mathrm{Cu}^{53}$ Modified silica nanoparticles were used to detect $\mathrm{Cu}^{2+}$ ions in solution, Figure 1c, by measuring the particles velocity (measured as the peak width) and the magnitudes, and occurrence of both the resistive, $\Delta_{\mathrm{ir}}$, and conductive pulses, $\Delta_{i c}$, Figures $1 \mathrm{~d}, \mathrm{e}$. The signal is specific to $\mathrm{Cu}^{2+}$ in the presence of other metal ions, and can be changed by adjusting the $\mathrm{pH}$ and ionic strength of the solution.

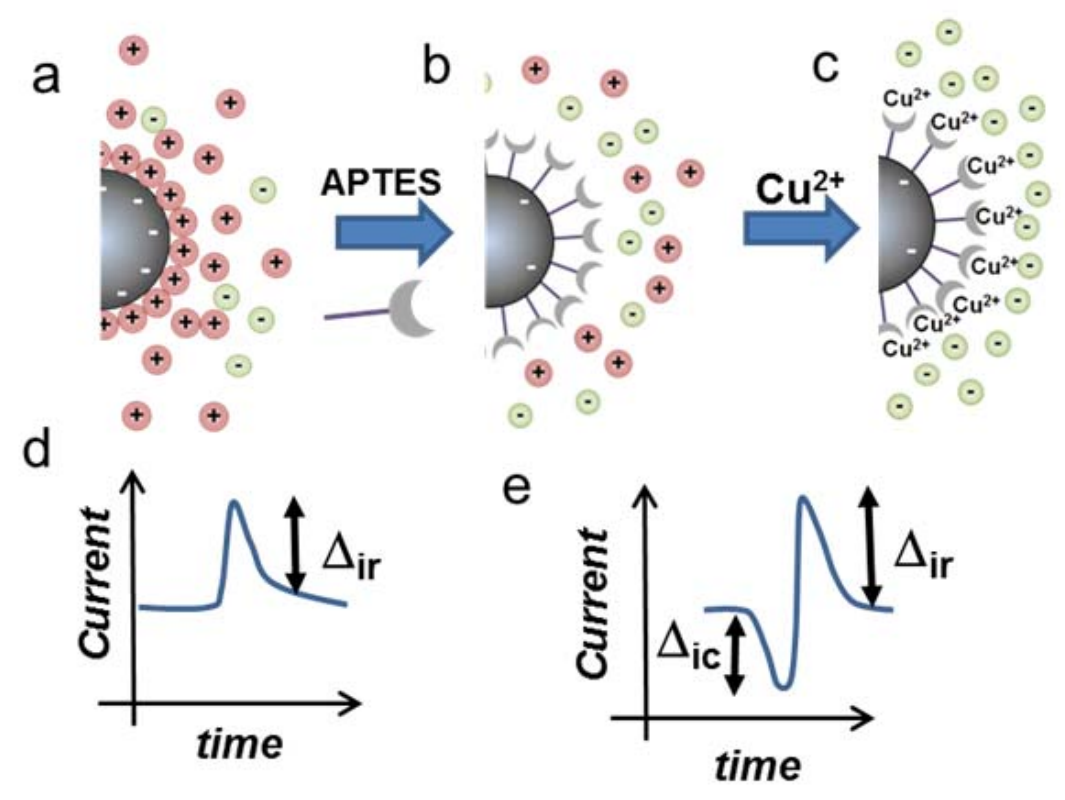

Figure 1. Schematic of the ion cloud a) around a $150 \mathrm{~nm}$ silica nanoparticle. b) A modified nanoparticle with APTES termed Si-APTES. c) Modified nanoparticle with $\mathrm{Cu}^{2+}$ copper bound to APTES, termed Si-APTES-Cu. d) Representation of a resistive pulse, e) Schematic of a double pulse, a conductive, $\Delta_{i c}$, followed by resistive pulse, $\Delta_{\text {ir. }}$

\section{Theory}

\section{Translocation velocity}

The frequency and velocity of particles passing through a nanopore can be shown to obey the following equations. The frequency of the pulses, $J$, is related to the concentration of the analyte, $C_{s},{ }^{54}$ and the particles velocity, $V_{p}$. 
$J=C_{s} \times V_{p}$

The velocity of the particle is a sum of the fluidic, $V_{F}$, electrophoretic, $V_{E}$, and electroosmotic, $V_{O}$, velocities, here diffusion is ignored as a small and insignificant contribution. ${ }^{54}$

$V_{p}=V_{F}+V_{E}+V_{O}$

$V_{p}$, can then be written as, ${ }^{55}$

$V_{p}=\varepsilon \frac{\zeta_{\text {particle }}-\zeta_{\text {pore }}}{\eta} E+\frac{Q_{p}}{A}$

Where, $\varepsilon$ and $\eta$ are the permittivity and the viscosity of the solution respectively, $\zeta_{\text {particle }}$ and $\zeta_{\text {pore }}$ are the zeta potentials of the particle and the channel surface respectively, $E$ is the electric field, $Q_{p}$ is the pressure driven volumetric fluid flow rate and $A$ is the cross sectional area of the pore. ${ }^{55}$ The relative velocity of the particle can then be calculated from the pulse width, Figure $S 1^{24}$. Multiple time points are recorded along the peak and are donated $\mathrm{T}_{0.90}, \mathrm{~T}_{0.80}, \mathrm{~T}_{0.70}$ etc., and the reciprocal of the average time from each point can be used to calculate the relative particle velocity ${ }^{24}$.

TRPS strategies have shown how the velocity of the particle can be used to determine its zeta potential. ${ }^{24,26}$ Here we stop short of calculating the actual zeta potential for two reasons. Firstly we cannot assume that the double layer thickness is short with respect to the particle diameter and pore opening and thus the particle velocity may be subject to electrophoretic retardation, and/or subject to a strong electro-osmotic flow, EOF, caused by the charged pore wall. Secondly, in each ionic strength and $\mathrm{pH}$ experiment the zeta potential of the pore wall would need to be known to calculate the zeta potential of the particle. ${ }^{24,56}$ To keep the method simple and applicable in further applications the actual zeta potential of the particle is not calculated and for further simplicity in the subsequent figures we use only one measurement to represent the particle speed which is $1 / T_{0.50}$.

In the case where the particle translocation causes a biphasic pule, the values for pulse magnitude, and $T_{0.5}$ for the resistive pulse are extracted from a base line from before and after the translocation event. Three examples of pulses, and the base line are given in Figure 2.
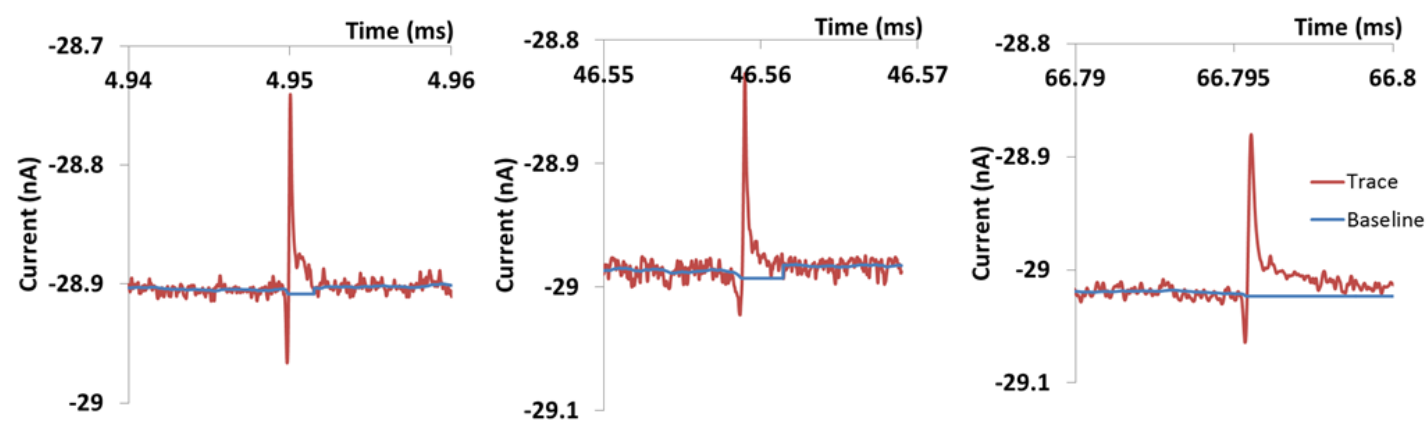

Figure 2. Current-time recording of a particle translocations. The blue line represents the baseline from which the pulse magnitudes are measured. 
If the pore charge, size, and fluid flow rates remain constant throughout the measurement, then equation 3 would predict that changes to the particles zeta potential, which is influenced by its surface charge and double layer structure, would result in a change in translocation times.

\section{Experimental}

\section{Materials and Methods}

Silica nanoparticles, SiNPs, (150 nm in diameter, $5 \%$ wt, 56799), (3aminopropyl)triethoxysilane (APTES, $\geq 98 \%$, A3648), magnesium (II) chloride $\left(\mathrm{MgCl}_{2}\right.$, powder, $\left.<200 \mu \mathrm{m}, 208337\right)$, copper (II) chloride dehydrate $\left(\mathrm{CuCl}_{2} .2 \mathrm{H}_{2} \mathrm{O}\right.$, reagent grade, 221783), iron (II) chloride tetrahydrate $\left(\mathrm{FeCl}_{2} .4 \mathrm{H}_{2} \mathrm{O}, 98 \%, 220299\right)$ were purchased from Sigma Aldrich, UK. Potassium chloride (KCl, >99\%, P/4240/60), potassium hydroxide $(\mathrm{KOH}, 0.1 \mathrm{M},>85 \%, \mathrm{P} / 5600 / 60)$, calcium chloride $\left(\mathrm{CaCl}_{2}\right.$, general purpose grade, $\mathrm{C} / 1400 / 60)$ and sodium chloride $(\mathrm{NaCl}$, analytical grade, $\mathrm{S} / 3160 / 60)$ were purchased from Fisher Scientific, UK. Hydrochloric acid $(\mathrm{HCl}$, $0.5 \mathrm{M}, 37 \%$ ) was purchased from VWR, UK. All reagents were used without further purification and all solutions were prepared in purified water with a resistance of $18.2 \mathrm{M} \Omega \mathrm{cm}$ (Maxima). Prior to analysis the $\mathrm{pH}$ of the solution was measured using a Mettler Toledo easy five $\mathrm{pH}$ meter with a Mettler Toledo InLab micro electrode.

\section{Tunable resistive pulse sensing}

All measurements were conducted using the qNano (Izon Sciences Ltd, NZ) combining tunable nanopores with data capture and analysis software, Izon Control Suite v.3.1. Analysis of the conductive pulses generated was performed using Clampfit 10 software. The lower fluid cell always contains the electrolyte $(75 \mu \mathrm{L})$ that the particle is suspended in and the upper fluid cell contains $40 \mu \mathrm{L}$ of sample. Prior to TRPS analysis, all samples were vortexed for $30 \mathrm{~s}$ and sonicated for 2 minutes. During each sample run, the system was washed by placing $40 \mu \mathrm{L}$ of the run electrolyte into the upper fluid cell several times with various pressures applied to ensure there were no residual particles remaining and therefore no cross contamination between samples. A detailed description of such a tunable resistive pulse sensing device can be found in Willmott et al. ${ }^{31}$ and Vogel et al. ${ }^{57}$. In all experiments we placed the particles in the upper fluid cell (on the side of the pore with the smallest opening).

\section{Silica Nanoparticle Modification}

The SiNP's $(5 \mathrm{~mL})$ were centrifuged for 10 minutes at 10,000 rpm and washed with acetone $(5 \mathrm{~mL})$. The wash step was repeated and the particles were suspended in acetone $(5 \mathrm{~mL})$. The SiNP solution was heated to $60^{\circ} \mathrm{C}$ in a round bottom flask and APTES $(1 \mathrm{~mL})$ added to the reaction and then refluxed for $2 \mathrm{hr}$. The solution was filtered under vacuum using a sintered glass crucible and the nanoparticles were washed with excess acetone before being left to air dry. This yielded APTES modified silica nanoparticles, Si-APTES. The presence of the APTES on the surface was confirmed using FT-IR (PerkinElmer).

\section{Calculating the particle velocities}


The method always used the resistive pulse to calculate the relative velocity, and identifies the point of greatest resistance in the signal trace (the resistive blockade peak), using a base line as illustrated in Figure 2. The same method is used for the biphasic pulses. For each blockade, the time at which the peak occurs is defined as T1.0 (time at $100 \%$ of peak magnitude) and the maximum magnitude of the pulse (relative to the local baseline resistance) is recorded as dRmax (see Figure s1). In the example shown in Figure s1, 4 sections, $60 \%, 50 \%, 40 \%$, and $30 \%$ of $\mathrm{dRmax}$ are displayed. The duration from T1.0 to each of these sections is defined at T0.60, T0.50, T0.40 and T0.30. When the proportional blockade magnitude is equal for any given particles (small or large), these particles are at the same position within the pore. Hence the relative magnitude is an indicator of the particles position within the pore.

\section{Metal Ion Extraction}

A copper (II) solution (1000 ppm) was prepared from $\mathrm{CuCl}_{2} \cdot \mathrm{H}_{2} \mathrm{O}$. The solution was diluted further to give a range of copper concentrations $(0.1-100 \mathrm{ppm})$. A range of metal ion solutions were prepared from $\mathrm{MgCl}_{2}, \mathrm{FeCl}_{2} .4 \mathrm{H}_{2} \mathrm{O}, \mathrm{CaCl}_{2}$ and $\mathrm{NaCl}$ at a 10 ppm concentration. A solution containing magnesium (II), iron (II), calcium (II), sodium (I) and copper (II), with each metal ion at a 10 ppm concentration, was prepared. To the solutions, Si-APTES particles were added to give an end concentration of the particles in the solution of $1 \mathrm{mgml}^{-1}$. The solutions were vortexed and sonicated to ensure the particles were fully dispersed and left for $24 \mathrm{hr}$ before analysis. The $\mathrm{pH}$ of copper solutions (10 ppm) were altered to $3.4,5.2$ and 7.0 , the modified particles were incubated in the three solutions for $24 \mathrm{hr}$ before analysis. To a range of copper (II) solutions, the modified particles were incubated for a shorter period of time and analysed after $5 \mathrm{~min}$. $\mathrm{pH}$ measurements on each solution were taken before and after the metal extraction.

\section{SEM Analysis}

Samples were placed on aluminium stubs and the electrolyte evaporated to leave a solid. EDX analysis was performed using a JOEL 7100F Field Emission Scanning Electron Microscope.

\section{Results and discussion}

The speed at which the particles traverse the pore can be related to the surface charge, as described above. This is dominated by the negative silica surface; or in later experiments by the amine terminated ligand; and any additional charge acquired through the binding of the metal ions. Firstly the purchased Si particles were placed into the instrument. These particles have a net negative charge at $\mathrm{pH} 7$, and travel towards the anode. Figure $3 a$ shows the average velocities for silica particles across a range of different electrolyte concentrations. The particle velocity is denoted as $\mathrm{ms}^{-1}$ and is the time taken for the particle to travel $50 \%$ of the way through the sensing zone of the pore. As the concentration of the supporting electrolyte is lowered the particle velocity for the bare silica particles increases from $2 \mathrm{~ms}^{-1}$ to $\sim 8.2 \mathrm{~ms}^{-1}$ in $100 \mathrm{mM}$ and $2 \mathrm{mM} \mathrm{KCl}$ respectively, Figure $3 a$. 


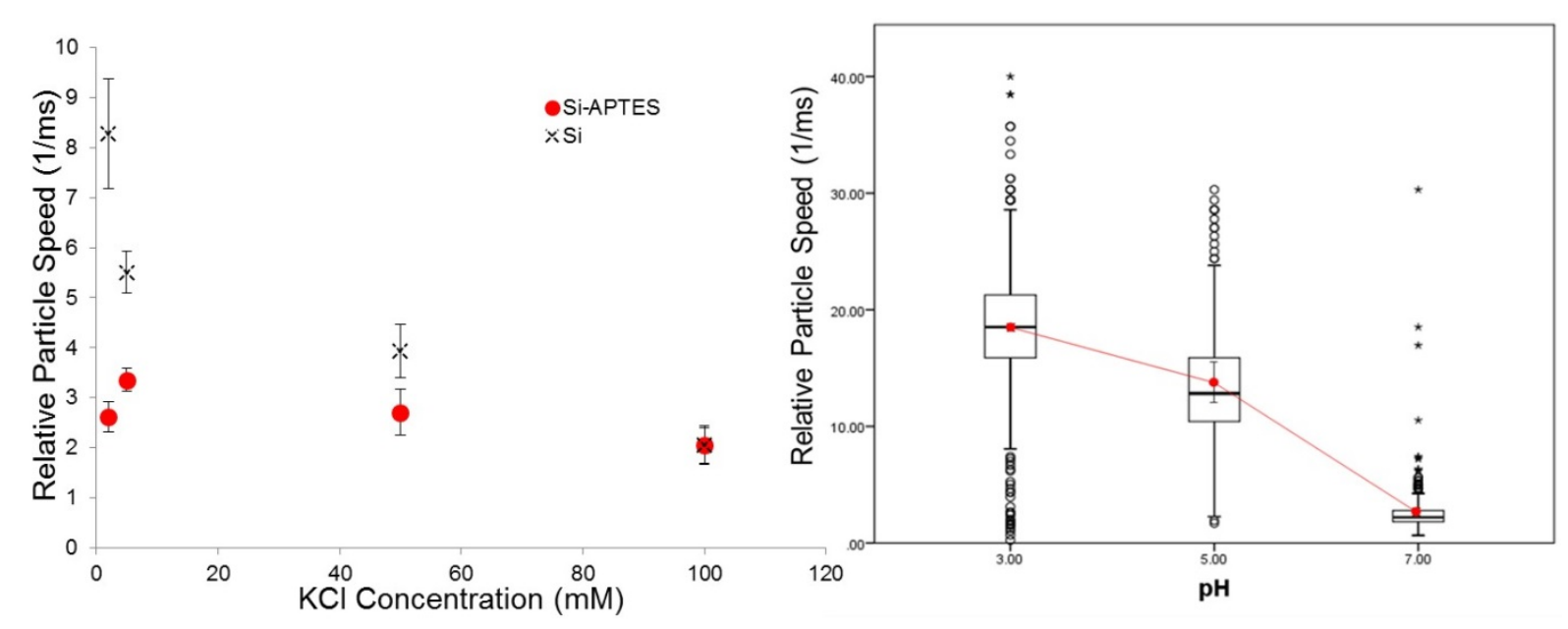

Figure 3. a) Change in translocation velocity for silica nanoparticle (crosses) with increasing $\mathrm{KCl}$ concentration. Solution $\mathrm{pH} 7.0 \pm 0.2 \mathrm{pH}$ using a NP2O0 pore, $47 \mathrm{~mm}$ stretch. b) Boxplot of Si-APTES particle speed as a function of electrolyte $\mathrm{pH}, \mathrm{pH} 7, \mathrm{pH} 5$ and $\mathrm{pH}$ 3. Measured in $2 \mathrm{mM} \mathrm{KCl}$. Samples run on a NP200 pore, $47 \mathrm{~mm}$ stretch and $-3.18 \mathrm{~V}$. Red circles represent the mean velocity with error bars representing the standard deviation from three experiments.

The measured velocity is related to the particles electrophoretic mobility and is a function of the double layer structure as described by the Henry relationship. Changes to the ionic strength and $\mathrm{pH}$ can then affect the measured velocity and Figure $3 a$ shows the effect of reducing the ionic strength of the electrolyte, and thus the shielding of the charge on the particles surface, by increasing the double layer thickness. Varying the $\mathrm{pH}$ had little effect, as the Si surface charge density remains constant and negatively charged over the $\mathrm{pH}$ range of 3-7, Figure S2. The addition of the APTES molecule onto the particle surface was firstly checked by measuring the speed and direction of translocation of the particles through the pore, and secondly with FT-IR using the presence of the primary amine at $1635 \mathrm{~cm}^{-1}$ (Figure S3).

Upon having their surfaces changed with the APTES the particles appear to have a small net positive surface charge at $\mathrm{pH} 7$, likely to be due to the protonation of the amine, and travel to the cathode. When the anode was placed inside the pore no translocation events were observed. In contrast to the bare Si beads, the velocity of the APTES modified beads remains unchanged across the different ionic strengths going from $2 \mathrm{~ms}^{-1}$ to $2.8 \mathrm{~ms}^{-1}$ in $100 \mathrm{mM}$ and $2 \mathrm{mM} \mathrm{KCl}$ respectively again attributed to the low charge on the APTEs ligand. As expected the charge on the primary amine can be controlled by varying the $\mathrm{pH}$, shown in Figure $3 \mathrm{~b}$. The $\mathrm{pK}_{\mathrm{a}}$ of APTES has been reported to be between $3.9-6.55^{58,59}$ therefor at an acidic $\mathrm{pH}$, the number of protonated amines increases, increasing the velocity of the particle. This is in contrast to the behaviour of silica particles whose speed decreases in acidic conditions Figure S2.

A benefit of the TRPS system is the Particle-by-Particle analysis. Figure 3c provides the distribution of velocities for the APTES modified particles. As well as observing a shift in the mean velocity from $2 \mathrm{~ms}^{-1}$ to $18 \mathrm{~ms}^{-1}$ in solution $\mathrm{pH} 7$ and 3 respectively, a broadening and change in the distribution is also observed with median skewness going from $0.57,0.33$ and -0.05 for $\mathrm{pH} 7,5$ and 3 respectively. This distribution of the data set represents the ability to resolve the difference in velocities of the particles 
that arise from the non-uniform distribution of APTES ligands on the particles surface, i.e. it is not expected that each particle will have the same number of APTES ligands on their surface and therefore the same velocity. To demonstrate that the observed velocities are dominated by the particles charge and not the electroosmotic flow within the pore, current voltage scans across a range of $\mathrm{pH}$ for $2 \mathrm{mM} \mathrm{KCl}$ are presented in Figure S4. Whilst some current rectification is observed here, it is likely to be a small contributing factor in the experiment and on a much smaller scale then seen in other TRPS systems. ${ }^{40}$

\section{Detection of Copper via Translocation speed.}

The APTES modified particles were then incubated in $10 \mathrm{ppm} \mathrm{Cu}^{2+}$ at three different $\mathrm{pH}$ values $(7.0,5.2$ and 3.4$)$ for 24 hours. Within 5 min a colour change (white blue) was present on the Si-APTES nanoparticles at $\mathrm{pH} \sim 7$ and $\sim 5$, whereas no colour change was observed at $\mathrm{pH} \sim 3$. Following the incubation, an aliquot of the particles were removed and diluted into a $\mathrm{KCl}$ supporting electrolyte solution. The velocities, recorded from the resistive pulse, of the particles across a range of electrolyte concentrations at $\mathrm{pH} 7$ are shown in Figure $4 \mathrm{a}$, (for comparison the SiAPTES data in Figure $3 a$ is reproduced). In contrast to the previous results in Figure 3 , as the electrolyte concentration decreases the velocity of the particles increase. The increase in velocity is attributed to the $\mathrm{Cu}^{2+}$ binding to the particles surface, increasing the particles surface charge. The translocation velocity of the Si-APTES$\mathrm{Cu}$ particle's at different $\mathrm{pH}$ values is given in Figure $\mathrm{S} 5$. As the $\mathrm{pH}$ is lowered the difference between the velocities of the particles incubated with $\mathrm{Cu}^{2+}$ and the blank (i.e. no $\mathrm{Cu}^{2+}$ ) becomes smaller. The reason for this is attributed to the amine groups being protonated, inhibiting the interaction between the amine and $\mathrm{Cu}^{2+}$ ion.

To verify that the particles were binding to the $\mathrm{Cu}^{2+}$, the concentration of the copper remaining in solution at $\mathrm{pH} 7$, was analysed using ICP-OES, Figure S6. EDX analysis on the particles was also performed shown in Figure 4b, c and d (for a high res image of the initial particles see supplementary Figure S7). In cases for $\mathrm{pH} 5$ and 7 , copper was detected on the particles surface, whilst no copper was observed via EDX at $\mathrm{pH} 3$. 


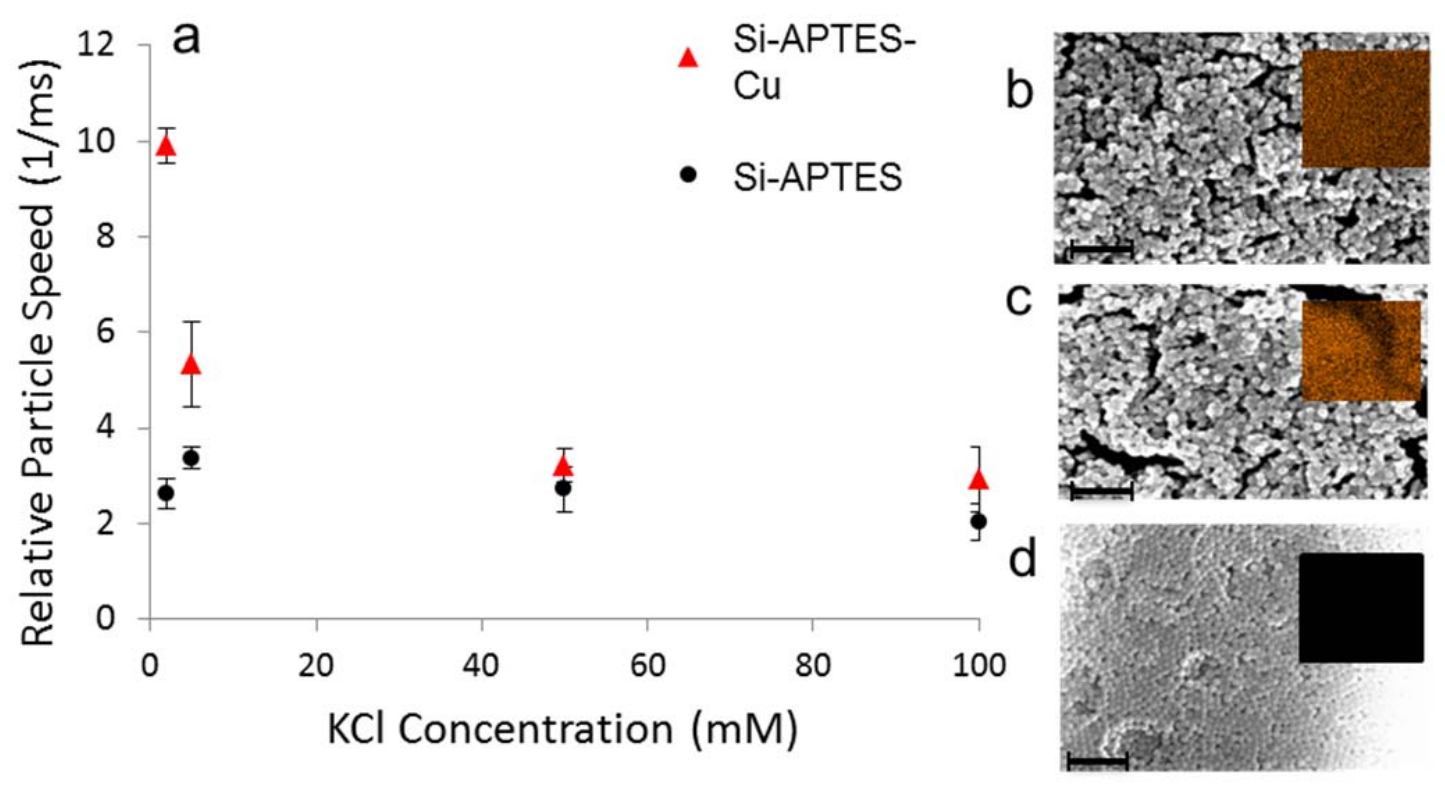

Figure 4. a) Si-APTES nanoparticles incubated with $10 \mathrm{ppm}$ CU (II) for $24 \mathrm{hr}$. Samples run on a NP200 pore, $\mathrm{pH} \sim 7$, stretch $46.50 \mathrm{~mm}$. Error bars represent the standard deviation of three experiments. $b$-d) SEM images of the particles, and inserted in each figure is the EDX analysis where the presence of copper is indicated in orange, samples obtained at b) $\mathrm{pH} 7.0$ c) 5.2), and d) 3.4. Scale bar = $1 \mu M$.

\section{Detection of Copper via pulse shape.}

In addition to the change in particle velocity, the wave shape of translocation events was investigated. The magnitudes of the resistive and conductive pulse are a function of the particles surface charge. Changes in their size and shape can be seen as the nanoparticle surface is altered, even if the particle size does not change. In low ionic strength $(<50 \mathrm{mM} \mathrm{KCl})$ biphasic pulse behaviour has previously been reported in both conventional and tunable RPS. ${ }^{48,60,61}$ It is thought that the conductive pulse arises from a charged particle passing through an area of ion accumulation, the nanoparticles counter ions increases the ionic concentration within the pore, which is recorded as an increase in conductivity before the volume exclusion generates a resistive pulse. ${ }^{48}$ It has been shown that the magnitude of the conductive pulse is dependent on the voltage, applied pressure, pore surface charge and the charge of the translocating particle. ${ }^{48}$ The resistive pulse magnitude is also a function of nanoparticle charge.$^{28,48}$ To summarise the expected results based upon theory, at negative potentials, a negatively charged particle passing through a negatively charged pore will generate a conductive pulse before the resistive pulse, Figure 1e. A positively charged particle, under the same conditions will only generate a resistive pulse but with a greater magnitude compared to the resistive pulse generated from the negatively charged particle of the same size. 


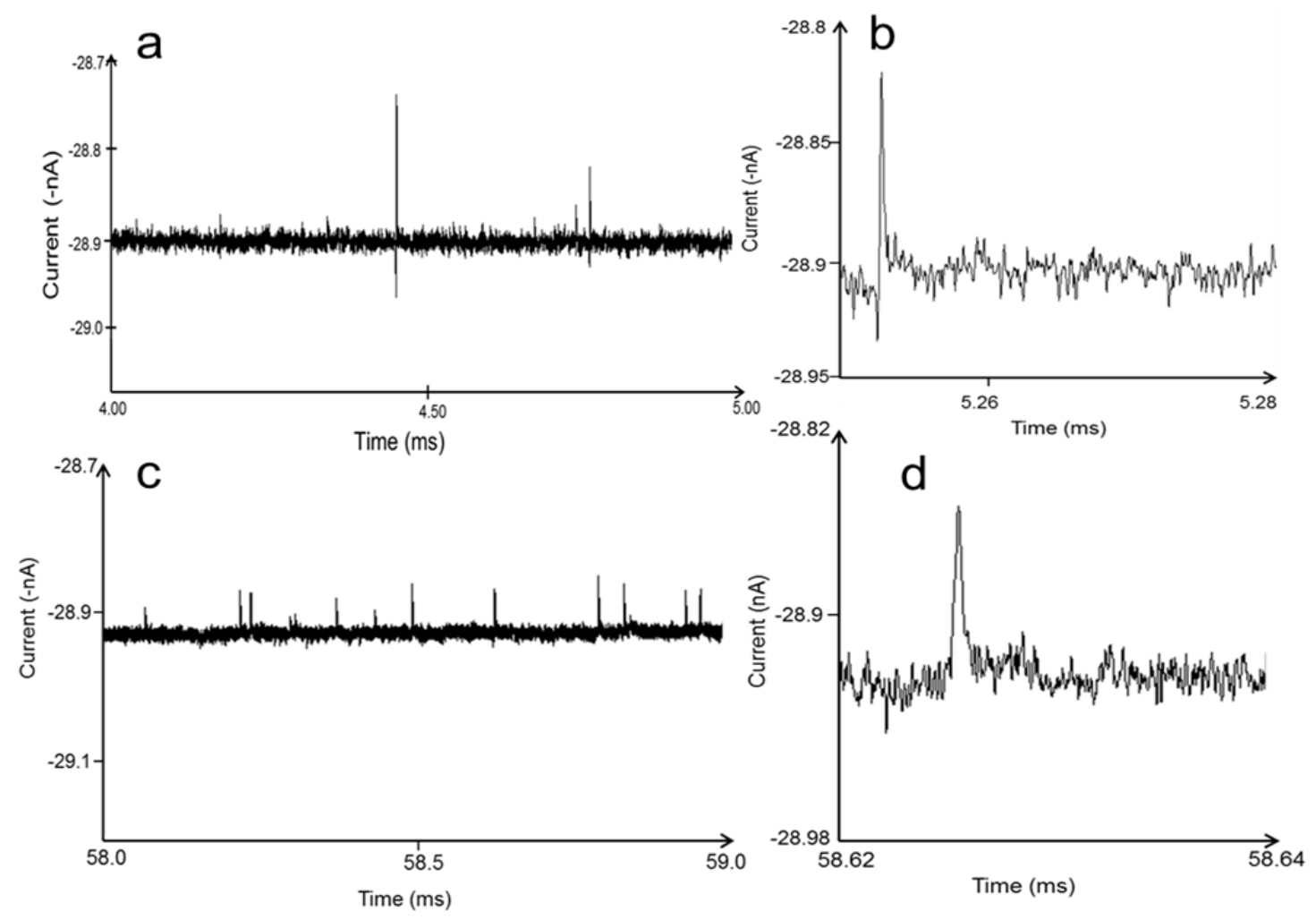

Figure 5. Current- time recordings of particle translocations measured in $2 \mathrm{mM} \mathrm{KCl}$ pH 7. a) Si-APTES translocation, $-3.94 \mathrm{~V}$, b) Magnified pulse from a. c) Si-APTESCu particle translocation, $-3.94 \mathrm{~V}$. d) Magnified single particle translocation from $\mathrm{c}$.

For APTES modified particles both a small conductive and larger resistive pulse was observed at both $\mathrm{pH} 7$ and 5 , examples of $\mathrm{pH} 7$ shown in Figure 5 . At the higher $\mathrm{pH}$ the pore surface is negatively charged, and the conductive pulse magnitude decreases with increasing positive charge on the particle, i.e. the binding of the $\mathrm{Cu}^{2+}$ ion to the APTES ligand. At pH 5 and the presence of $\mathrm{Cu}^{2+}$, a small $\Delta_{\text {ic }}$ was seen by the user, but the software was unable to distinguish it from the background noise. In addition to the $\Delta_{\text {ic }}$ decreasing the $\Delta_{\text {ir }}$ increased, the values for the pulse magnitudes are given on Figure 6 . Changes in pulse magnitude can arise from particles aggregating or of axial transport ${ }^{62}$. Here, the particles show no significant aggregation when the $\mathrm{Cu}^{2+}$ ion binds, shown in Supplementary Figure S8. Of axial particle trajectory through the pore has also been shown to effect the measured particle blockade, however our results are averaged over hundreds of particles and are relative across a concentration range for the same pore, and should therefore be accounted for within the study. ${ }^{62}$ We conclude then that the changes in magnitude are due to the particles counter ions either traversing the pore or being depleted from the pore interior due to the pore surface charge.

The change in pulse shape offers an extra verification that the $\mathrm{Cu}$ has bound to the particle. When the copper binds to the nanoparticle at $\mathrm{pH} 5$, the particles increased positive charge results in changes in the waveshape. A Si-APTES particle creates a biphasic pulse as it traverses the pore. When copper is bound to the particle, only a single resistive pulse is generated. The resistive pulse magnitude increases when copper is bound, Figure 6a. This is explained by the enhanced depletion effect 
generated by the conical pore and its current rectification properties. This effect interacts with the ion cloud around the positively charged particle.

At $\mathrm{pH} 5$, no significant velocity differences between Si-APTES and Si-APTES-Cu ${ }^{2+}$ are observed, Figure S5, but measuring the differences in pulse magnitudes allows the changes of the nanoparticle surface to be detected. The same trend for pulse magnitude is observed for particles analysed in $\mathrm{pH} \mathrm{7,} \mathrm{Figure} 6 \mathrm{~b}$. The conductive pulse magnitude decreases and the resistive pulse magnitude increases when copper binds to the particle. At $\mathrm{pH} \mathrm{3}$, the resistive and conductive pulses did not significantly change, shown in supplementary Figure S9. This is due to no copper being bound at the low $\mathrm{pH}$, which is also shown by no change in particle velocity. It's also interesting to note the error bars in Figure 6, that show the variation in conductive peak size is much smaller than that of the resistive peaks. It may be that the conductive peaks are less effected by off axial translocations.

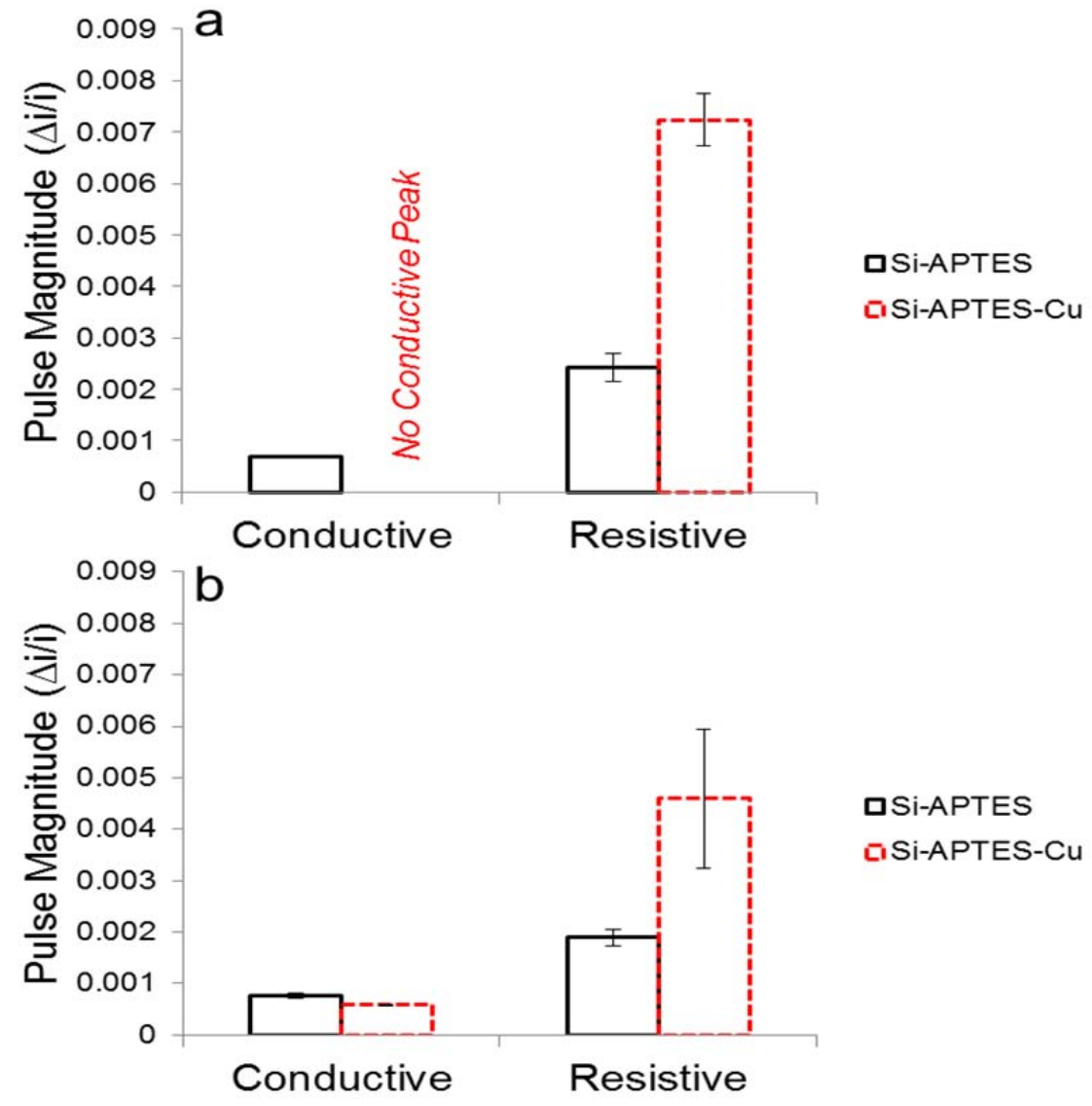

Figure 6. a) Conductive and resistive pulse magnitudes recorded for particles with and without copper bound at $2 \mathrm{mM} \mathrm{pH} \mathrm{5,-3.94} \mathrm{V.} \mathrm{No} \mathrm{conductive} \mathrm{pulse} \mathrm{generated} \mathrm{from} \mathrm{Si-}$ APTES-Cu particles. b) Conductive pulse and resistive pulse magnitudes recorded for particles with and without copper bound at $2 \mathrm{mM} \mathrm{pH} \mathrm{7,-3.94} \mathrm{V.} \mathrm{Dashed} \mathrm{black} \mathrm{lines}$ represent Si-APTES and red solid lines represent Si-APTES-Cu particles. Error bars represent one standard deviation from the mean for three samples. 
Whilst the pulse shape offers an ability to determine the presence of the metal ion, the simplest value to extract was the velocity, with the largest change in velocity being observed in $2 \mathrm{mM} \mathrm{KCl} \mathrm{pH} \sim 7$. Experiments at this electrolyte concentration makes counting a significant number of particles $(>300)$ difficult, and time consuming (this is due to low particle count and high background noise). We wished to produce a technique that is both sensitive and easy to use by less experienced users in the hope it is adopted as a sensor. Thus results presented here after are analysed in $5 \mathrm{mM}$. At $5 \mathrm{mM} \mathrm{KCl}$ an acceptable particle count $(>300 / 5 \mathrm{~min})$ can be obtained and allows the velocity between Si-APTES and Si-APTES- $\mathrm{Cu}^{2+}$ to be distinguished, see Figure 3 .

To demonstrate a quantitative experiment; Si-APTES particles were placed into a several concentrations of solutions of $\mathrm{Cu}^{2+}$ at $\mathrm{pH}$ 7. A Blank, i.e. Si-APTES particles that had not been exposed to $\mathrm{Cu}^{2+}$, were analysed for comparison. Below $1 \mathrm{ppm}$, no significant difference was observed, however above $1 \mathrm{ppm}$ a clear calibration curve can be created. All experiments thus far have utilised a $24 \mathrm{hr}$ incubation period, and this time period is impractical for field measurements. To ascertain the speed in which the signal and $\mathrm{Cu}^{2+}$ could be measured the incubation time was decreased to 5 minutes, Figure $\mathrm{S} 10$, the incubated particles were analysed in $\mathrm{KCl}(5 \mathrm{mM}, \mathrm{pH} 7.0)$. Again a "blank" was analysed for comparison. Particles incubated for the shorter period of time in 10 and 100 ppm solutions showed a significant increase in speed in comparison to the blank. The longer incubation time of 24 hours did not significantly increase the velocity of the particles suggesting an equilibrium was established quickly.

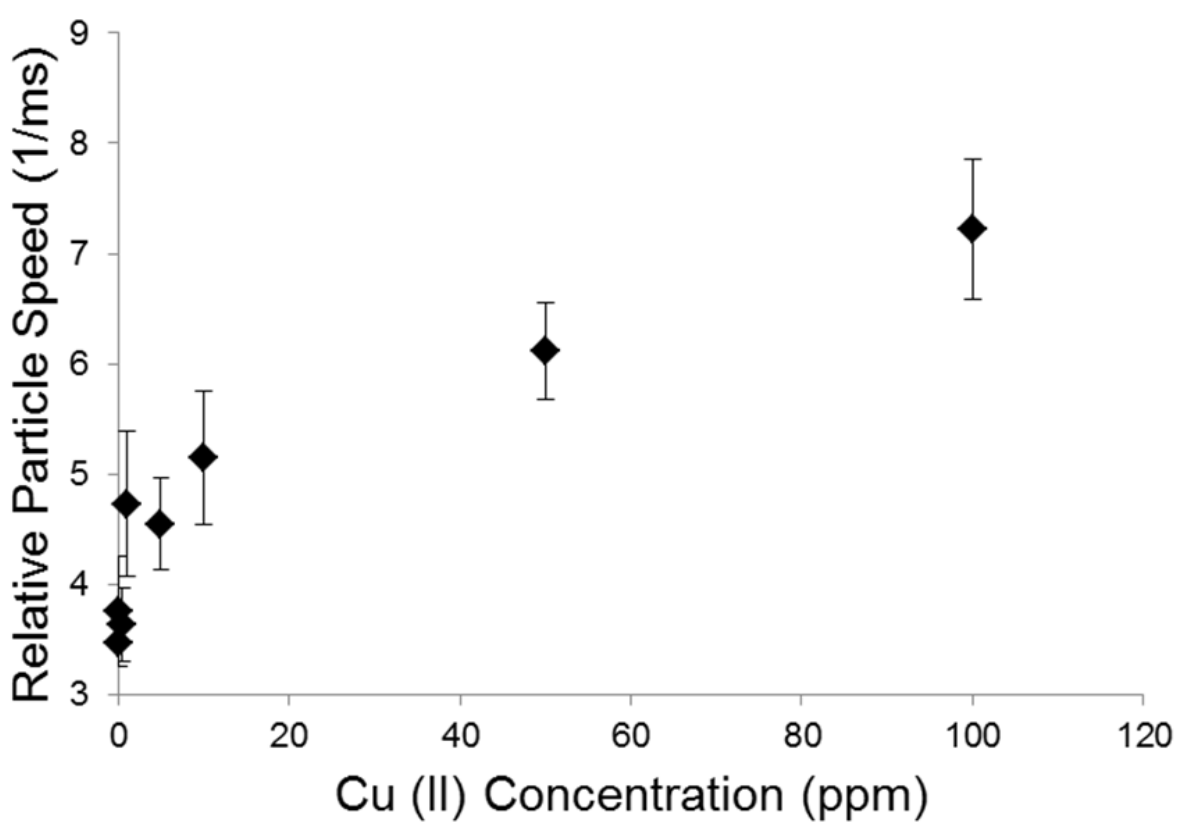

Figure 7. Concentration Assay: Si-APTES nanoparticles incubated in different Cu (II) concentration solutions for $24 \mathrm{hr}$, particle speed was analysed in $5 \mathrm{mM} \mathrm{KCl} \mathrm{pH} \mathrm{7.0.}$

To further test the selectivity of the APTES modified silica towards $\mathrm{Cu}^{2+}$, the modified nanoparticles were incubated in a series of different metal ions $\left(\mathrm{Mg}^{2+}, \mathrm{Na}^{+}, \mathrm{Ca}^{2+}\right.$, $\mathrm{Fe}^{2+}$ ) and also a solution containing a mixture of the above with $\mathrm{Cu}^{2+}$ presented in Figure 8 . The velocities of the particles were measured after $24 \mathrm{hr}$ in each solution. 
While all metals showed an increase in velocity from the blank, the particles incubated with copper gave the largest change from 3.3 to $7.4 \mathrm{~ms}^{-1}$. The particles incubated in the mix of metals show a similar increase in velocity to the copper system, suggesting the Si-APTES particles show preferential binding to the copper (II) ions. To verify this result the concentration of copper remaining in solution was measured by ICP-OES (Supplementary Figure S11). When no competing metal ions were present, the Si-APTES particle removed $77.1 \%$ of the $\mathrm{Cu}^{2+}$ ions over a $24 \mathrm{hr}$ period. With competing metal ions, the Si-APTES particles removed $72.6 \%$ of the $\mathrm{Cu}^{2+}$ ions.

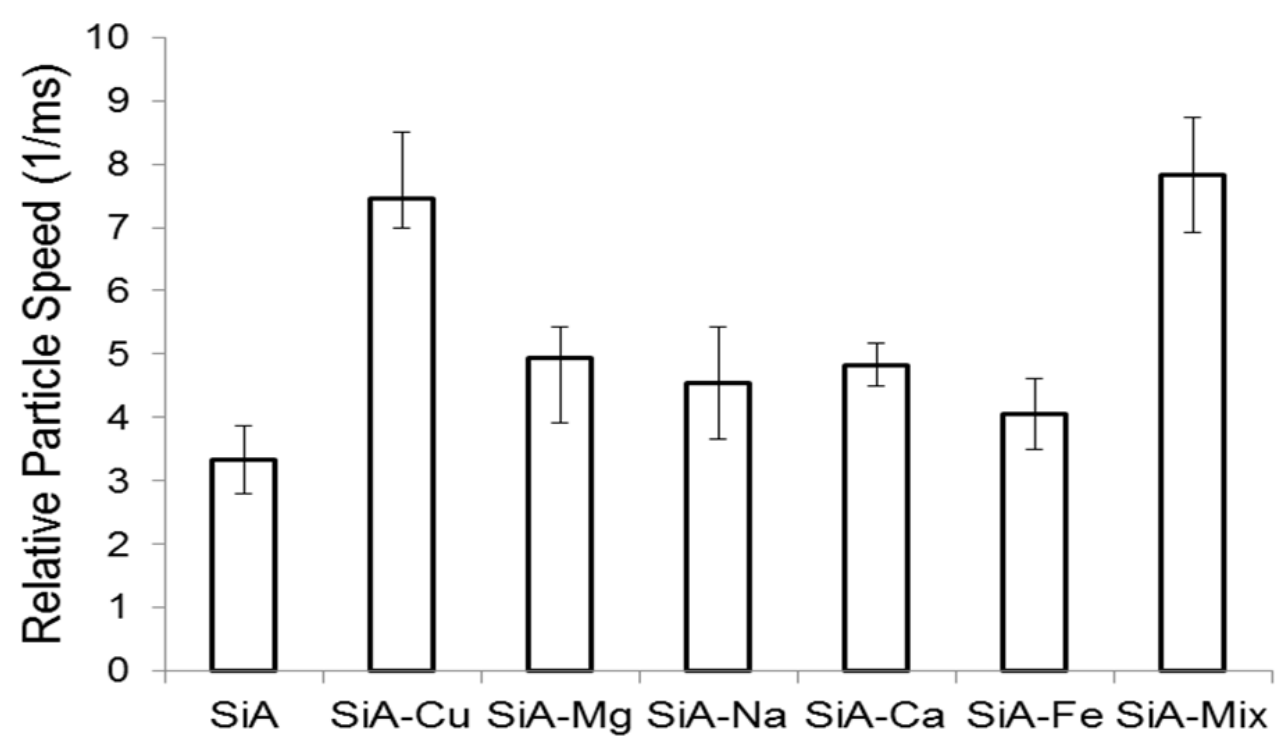

Figure 8. Selectivity of the Si-APTES nanoparticles for $\mathrm{Cu}^{2+}$ compared to $\mathrm{Mg}^{2+}, \mathrm{Na}^{+}, \mathrm{Ca}^{2+}$, $\mathrm{Fe}^{2+}$ and in the presence of the four metal ions (each metal ion at $10 \mathrm{ppm}$ ), Error bars represent one standard deviation from the mean of three repeats, Run on a NP200 pore, $47.00 \mathrm{~mm}$ and $-1.60 \mathrm{~V}$.

\section{Conclusions}

In summary we present a simple and rapid method to use the surface charge of nanoparticle for measuring heavy metal ions in solution. We demonstrate how the velocity of the particle changes over a range of ionic strengths and $\mathrm{pH}$ due to nanoparticle surface charge. The biphasic pulse behaviour of the particle translocating through the pore can also be used to monitor changes on the nanoparticle surface, and infer the presence of ions within the particles double layer. This method allows the quantitative measurement of metal ions in solution in less than 10 minutes. The method is adaptable to a series of metals via the modification of the $\mathrm{Si}$ surface, and as it is capable of running across a range of $\mathrm{pH}$ and ionic strengths the technique is suitable for biological and environmental studies. 


\section{Acknowledgements}

The work was supported by the European Commission for Research (PCIG11-GA2012-321836 Nano4Bio). The authors would also like to thank Dr James Holt for his help with elemental analysis.

\section{Notes and references}

A. Joshi and T. C. Nagaiah, RSC Adv., 2015, 5, 105119-105127.

2 Z. A. Alothman, E. Yilmaz, M. A. Habila, I. H. Alsohaimi, A. M. Aldawsari, N. M. AL-Harbi and M. Soylak, RSC Adv., 2015, 5, 106905-106911.

P. Ma, F. Liang, Q. Diao, D. Wang, Q. Yang, D. Gao, D. Song and X. Wang, RSC Adv., 2015, 5, 32168-32174.

Y. Guo, F. Cao, X. Lei, L. Mang, S. Cheng and J. Song, Nanoscale, 2016, 8, 4852-4863.

Y. Ye, M. Lv, X. Zhang and Y. Zhang, RSC Adv., 2015, 5, 102311-102317.

A. A. Asgharinezhad, N. Jalilian, H. Ebrahimzadeh and Z. Panjali, RSC Adv., 2015, 5, 45510-45519.

P. I. Girginova, A. L. Daniel-da-Silva, C. B. Lopes, P. Figueira, M. Otero, V. S. Amaral, E. Pereira and T. Trindade, J. Colloid Interface Sci., 2010, 345, 234-40.

M. Behbahani, A. Aliakbari, M. M. Amini, A. S. Behbahani and F. Omidi, RSC Adv., 2015, 5, 68500-68509.

S. Venkateswarlu and M. Yoon, RSC Adv., 2015, 5, 65444-65453.

E. Yilmaz, R. M. Alosmanov and M. Soylak, RSC Adv., 2015, 5, 33801-33808.

Z. Wang, D. M. Fang, Q. Li, L. X. Zhang, R. Qian, Y. Zhu, H. Y. Qu and Y. P. Du, Anal. Chim. Acta, 2012, 725, 81-86.

S. Wu, N. Chandra Sekar, S. N. Tan, H. Xie and S. H. Ng, Anal. Methods, 2016, 8, 962-967.

T. L. Read, M. B. Joseph and J. V Macpherson, Chem. Commun., 2016, 52, 1863-1866.

X. Meng, Y. Xu, J. Liu, L. Sun and L. Shi, Anal. Methods, 2016, 8, 1044-1051.

L. Chen, T. Ji, L. Mu, Y. Shi, L. Brisbin, Z. Guo, M. a. Khan, D. P. Young and J. Zhu, RSC Adv., 2016, 6, 2259-2269.

P. Nath, R. K. Arun and N. Chanda, RSC Adv., 2015, 5, 69024-69031.

H. Bayley and C. R. Martin, Chem. Rev., 2000, 100, 2575-2594.

L. T. Sexton, L. P. Horne and C. R. Martin, Mol. Biosyst., 2007, 3, 667-685.

D. Kozak, W. Anderson, R. Vogel and M. Trau, Nano Today, 6, 531-545.

L. Luo, S. R. German, W.-J. Lan, D. a Holden, T. L. Mega and H. S. White, Annu. Rev. Anal. Chem., 2014, 7, 513-35.

Z. Siwy, L. Trofin, P. Kohli, L. A. Baker, C. Trautmann and C. R. Martin, J. Am. Chem. Soc., 2005, 127, 5000-5001.

W.-J. Lan, D. A. Holden, J. Liu and H. S. White, J. Phys. Chem. C, 2011, 115, 18445-18452.

Y. Qiu, I. Vlassiouk, P. Hinkle, M. E. Toimil-Molares, A. J. Levine and Z. S. Siwy, ACS Nano, 2016, 10, 3509-3517

E. L. C. J. Blundell, R. Vogel and M. Platt, Langmuir, 2016, 32, 1082-1090.

N. Arjmandi, W. Van Roy, L. Lagae and G. Borghs, Anal. Chem., 2012, 84, 8490-8496.

J. A. Eldridge, G. R. Willmott, W. Anderson and R. Vogel, J. Colloid Interface Sci., 2014, 429, 45-52.

D. Kozak, W. Anderson, R. Vogel, S. Chen, F. Antaw and M. Trau, ACS Nano, 2012, 6, 6990-6997.

Y. Qiu, I. Vlassiouk, Y. Chen and Z. S. Siwy, Anal. Chem., 2016, 88, 4917-4925

G. Wang, Q. Zhao, X. Kang and X. Guan, J. Phys. Chem. B, 2013, 117, 4763-4769.

G. Wang, L. Wang, Y. Han, S. Zhou and X. Guan, Biosens. Bioelectron., 2014, 53, 453-458.

G. R. Willmott, R. Vogel, S. S. C. Yu, L. G. Groenewegen, G. S. Roberts, D. Kozak, W. Anderson and M. Trau, J. Phys. Condens. Matter, 2010, 22, 454116. 
E. R. Billinge, J. Muzard and M. Platt, Nanomater. Nanosci., 2013, 1, 1.

E. R. Billinge and M. Platt, Anal. Methods, 2015, 7, 8534-8538.

M. Platt, G. R. Willmott and G. U. Lee, Small, 2012, 8, 2436-2444.

G. R. Willmott, M. Platt and G. U. Lee, Biomicrofluidics, 2012, 6, 14103-14115.

E. Weatherall and G. R. Willmott, Analyst, 2015, 140, 3318-3334.

E. L. C. J. Blundell, L. J. Mayne, E. R. Billinge and M. Platt, Anal. Methods,7, 2015, 7055-7066

E. L. C. J. Blundell, L. J. Mayne, S. D. R. Christie and M. Platt, Faraday Discuss., 2016, DOI: 10.1039/C6FD00072J

Z. Siwy, E. Heins, C. C. Harrell, P. Kohli and C. R. Martin, J. Am. Chem. Soc., 2004, 126, 10850-10851.

Z. S. Siwy, Adv. Funct. Mater., 2006, 16, 735-746.

W.-J. Lan, D. A. Holden, B. Zhang and H. S. White, Anal. Chem., 2011, 83, 3840-3847.

N. Sa and L. a. Baker, J. Am. Chem. Soc., 2011, 133, 10398-10401.

Z. Siwy, E. Heins, C. C. Harrell, P. Kohli and C. R. Martin, J. Am. Chem. Soc., 2004, 126, 10850-10851.

J.-Y. Jung, P. Joshi, L. Petrossian, T. J. Thornton and J. D. Posner, Anal. Chem., 2009, 81, 3128-33.

R. Gao, Y. Ying, B. Yan, P. Iqbal and J. A. Preece, Microchimica Acta, 2016,183, 491-495.

W. J. Lan, C. Kubeil, J. W. Xiong, A. Bund and H. S. White, J. Phys. Chem. C, 2014, 118, $2726-2734$.

Y. Qiu, C.-Y. Lin, P. Hinkle, T. S. Plett, C. Yang, J. Varghese Chacko, M. a Digman, L.-H. Yeh, J.-P. Hsu and Z. S. Siwy, ACS Nano, 2016.

World Health Organisation, Copper in Drinking Water, 2004.

A. W. Foster, D. Osman and N. J. Robinson, J. Biol. Chem., 2014, 289, 28095-103.

G. J. Brewer, Clin. Neurophysiol., 2010, 121, 459-460.

J. D. Holt, PhD Thesis, Loughborough University, 2014.

R. W. DeBlois, C. P. Bean and R. K. . Wesley, J. Colloid Interface Sci., 1977, 61, 323-335.

J. A. Somerville, G. R. Willmott, J. Eldridge, M. Griffiths and K. M. McGrath, J. Colloid Interface Sci., 2013, 394, 243251.

P. Jin, H. Mukaibo, L. P. Home, G. W. Bishop and C. R. Martin, J. Am. Chem. Soc., 2010, 132, $2118-2119$.

R. Vogel, G. Willmott, D. Kozak, G. S. Roberts, W. Anderson, L. Groenewegen, B. Glossop, A. Barnett, A. Turner and M. Trau, Anal. Chem., 2011, 83, 3499-3506.

D. V Vezenov, A. Noy, L. F. Rozsnyai and C. M. Lieber, J. Am. Chem. Soc., 1997, 119, 2006-2015.

K. Van Der Maaden, K. Sliedregt, A. Kros, W. Jiskoot and J. Bouwstra, Langmuir, 2012, 28, 3403-3411.

J. Menestrina, C. Yang, M. Schiel, I. Vlassiouk and Z. S. Siwy, J. Phys. Chem. C, 2014, 118, 2391-2398.

E. Weatherall and G. R. Willmott, J. Phys. Chem. B, 2015, 119, 5328-5335.

E. Weatherall, P. Hauer, R. Vogel and G. R. Willmott, Anal. Chem., 2016, 88, 8648-8656 